\title{
EQUI-BIAXIAL TESTS FOR MECHANICAL CHARACTERIZATION OF HUMAN ACELLULAR DERMAL MATRICES THROUGH A CUSTOM-MADE BIAXIAL FIXTURE
}

\author{
MARA TERZINI $^{1}$, ALESSANDRA ALDIERI $^{1}$, CRISTINA BIGNARDI $^{1}$, ELISABETTA M. ZANETTI $^{2}$ \\ \& ALBERTO L. AUDENINO ${ }^{1}$ \\ ${ }^{1}$ Department of Mechanical and Aerospace Engineering, Politecnico di Torino, Italy \\ ${ }^{2}$ Department of Engineering, University of Perugia, Italy
}

\begin{abstract}
Human Acellular Dermal Matrices (HADMs) have recently been employed in reconstructive surgeries which involve high mechanical resistance prerequisites (e.g. rotator cuff tears repair, Achilles tendon augmentation, breast reconstruction procedure, hernia repair), having proved its non-immunogenic response and promising mechanical properties. Nevertheless, a thorough mechanical characterization is needed in order to test HADMs response when subjected to stress levels comparable to those experienced in vivo, to confirm its applicability to the above mentioned surgical fields and to provide precise indications to surgeons. HADMs specimens have undergone biaxial tests through a custom made biaxial fixture interfaced with a uniaxial universal testing machine. Stress-strain curves were evaluated from biaxial loads measured by two load cells, and the optical measure of the displacement of four markers located in central area of specimens. Comparisons among the native human reticular dermis and HADMs demonstrated the loss of mechanical strength caused by the decellularization process. Moreover, the specimens resulted, on average, less extensible in the medio-lateral direction (namely, along the Langer lines) than in the cranio-caudal direction, confirming the correlation of dermis mechanical response with collagen fibers orientation.

Keywords: human dermis, HADM, biaxial characterization, biaxial fixture, soft tissues material model identification.
\end{abstract}

\section{INTRODUCTION}

As the understating of the biological characteristics of dermis increases, the indications for its uses evolve and expand. HADMs (Human Acellular Dermal Matrices) were originally developed for use in the treatment of full-thickness burns, because of their not immunogenic response, handling, long shelf-life and expedite healing. Recently they found application in the repair of oral mucosal presenting very high success rates and avoiding any immunologic reactions and infections [1]. In fact, the last application responds to the same uncritical requisites in terms of mechanical response and patch integration.

The demand for high strength applications has started and is continuously raising in the last two decades. For instance, the use of HADMs in breast surgery began in 2001 [2] and the first published application of HADM use in breast reconstruction came in 2005; since its introduction, HADM use in breast surgery has quickly become one of the most popular applications. HADMs have also been proposed as a substitute of mesh materials in hernia repair surgery because of its lower rates of infection and breakdown. In this case, the structural strength of the hernia repair tissue is mandatory for a successful outcome. Applications such as the repair of rotator cuff tears [5]-[8] are even more demanding from the point of view of mechanical strength, as well as Achilles tendon augmentation. Here the dermal matrix is not only the support for tissue healing, but must accomplish a structural function, therefore its biomechanical behavior at high stress levels becomes very relevant. 
The human dermis exhibits a pronounced mechanical anisotropy because of its oriented fibrous structure; planar biaxial test is therefore recommended to fully characterize HADM properties at the expense of the complexity of the experimental set-up (e.g. two actuators, two load sensors, an optical measure of strain, etc.). Moreover, biaxial experiments on soft biological tissues are even more problematic due to the common small specimen size, the large specimen-to-specimen variability, the lack of homogeneity of deformation within the specimen, and artefacts produced by gripping. Dealing with the last aspect, in literature various solutions were adopted; in most of them the specimen is mounted in the device in trampoline-like fashion although the grasping mechanism vary from one author to another: the preponderant solutions make use of thin threads (suture material), clips and hooks.

Non-contact strain measurement is recommendable in order to avoid any mechanical bias on such a compliant material; an optical measure of strains can be obtained tracking the position of markers mounted in the upper specimen surface, as long as its visibility is guaranteed during the whole test. This measure relies on an imaging device able to generate a synchronized image acquisition, and the positioning of markers on the wet specimen substrate. The strain optical measure is generally performed on a central target region which must be sufficient small and located away from the specimen edges to be unaffected by gripping. In this central region, the stress-strain field can be considered to be homogenous. Actually, the bias produced by specimen fixtures may be different according to the gripping method: whenever the specimen ends are clamped, transversal displacements are prevented, while they are not so negligible in the case of soft tissue. Suturing can be a good alternative: it resets the chances of slippage, and is closer to reality where the allograft is placed via suture in vivo during a reconstructive surgery leading to point loads in the immediate postoperative.

The substantial anisotropy of soft biological tissues make it necessary to record not only a local distribution of strains on the specimen surface, but also load responses from the two orthogonal directions, without going through any indirect evaluation of forces and stresses.

The first investigators to develop [24] and utilize [25] planar biaxial testing for soft biological tissues were respectively Lanir and Fung [25]. They investigated the mechanical properties of the rabbit skin demonstrating its nonlinear and orthotropic stress-strain response and the dependence of the latter on the specimen anatomic orientation. Moreover, they developed

the most broadly used constitutive model for the biaxial response of skin. Since then, the biaxial characterization of soft tissues has been performed extensively on cardiac and artery tissues, with the aim of implementing constitutive models to carry out preclinical tests of intravascular devices, and the porcine meniscal. In many cases, the research outcome are model parameters obtained fitting a stress strain curve, obtained as a mean of curves acquired along two orthogonal axes. The assumption behind this procedure is that although the anisotropy of the tissue is obvious and confirmed by the biaxial tests, the response of the tissue along two orthogonal axes is no significantly different at physiological loads. This assumption does not hold for the HADM, since it undergoes high and unbalanced strains, which bring out the marked anisotropy of this tissue, both in vivo and in its clinical applications.

The aim of this work is to fill this gap presenting equi-biaxial experimental curves of decellularized human dermis. This mechanical characterization is aimed to a double aim: optimizing the chemical treatments required to decellularize the dermal matrix, and investigating the engineered tissue response at high stress levels, comparable to those experienced in vivo. 


\section{MATERIALS AND METHODS}

\subsection{Biaxial mechanical tests}

Dermis strips unfit for transplantation were used for this research after the approval of the Institutional Ethical Board of Azienda Ospedaliera Universitaria Città della Salute e della Scienza of Turin, Italy, (approved on January $23^{\text {rd }}, 2012$ with protocol number 0006730), and written informed consent was obtained from all study participants.

Large strips of dermis tissue, collected from the backs of three human donors (A, B and $\mathrm{C}$ ), were dissected along the cranio-caudal direction. They were then decellularized using an incubation in DMEM medium for 5 weeks. The treatment type and its duration were set according to the results of a previous optimization. Intact not decellularized human reticular dermis coming from the same donors was used as a control. Both the native human reticular dermis (Control) and HADM used for these experiments were preserved at $85 \%$ glycerol in a $4{ }^{\circ} \mathrm{C}$ refrigerator. The specimens were obtained by cutting out $30 \times 30 \mathrm{~mm}$ squares using a custom-made die cutter. A maximum of four specimens from each strip was obtained, except for the undecellularized strip (Control) coming from donor B, where two only specimens have been obtained due to its small size. The total number of specimens is summarized in Table 1.

The ML orientation has been indicated on each specimen by means of stitches sewed at the upper left vertex of the square. One stitch represented the first specimen, two stitches the second one, and so on (Fig. 1). The sewed stitches have been positioned so as to avoid interferences with the gripping of the specimen. This numbering is needed in order preserve the original orientation and location during measurement and washing procedures.

Before testing, dermis grafts were washed to remove all glycerol, dipping them sequentially in three different beakers filled with abundant saline solution $0.9 \%$ at $+37^{\circ} \mathrm{C}$ for more than three minutes each. The specimens thickness at rest was then acquired by means of photogrammetry (full-frame digital camera - Canon EOS 5D Mark II - with an autofocus lens for macro photography - Canon EF $100 \mathrm{~mm} \mathrm{f} / 2.8$ Macro USM) as an average of five measures evaluated by image analysis software ImageJ (National Institutes of Health, Bethesda, Maryland, U.S.). An average thickness equal to $2.43 \pm 0.46 \mathrm{~mm}$ (average $\pm \mathrm{std}$ ) was obtained. Samples were subjected to equi-biaxial tensile tests by means of a custom made biaxial testing device, designed by our group, as an accessory of the uniaxial tensile machine (Bose Electroforce ${ }^{\circledR} 3200$ ), taking advantage of the machine own actuator and data acquisition system, integrating the experimental set up with additional sensors. The device has been entirely realized through rapid prototyping methods (3D printer Stratasys uPrint SE Plus - Stratasys Inc., Edina, Minnesota, United States) greatly reducing the cost of materials and machining.

The conversion from uniaxial to biaxial motion is made possible by the presence of four commercial linear guideways (Hiwin ${ }^{\circledR} \mathrm{MGN}$ ) placed at $45^{\circ}$ with respect to the actuator axis. The operating principle is the following: when the uniaxial machine crosshead goes up, the device upper crosshead moves with a velocity " $v$ " whereas the lower crosshead remains motionless. Concurrently, the lateral links move up with a velocity of "v/2" and simultaneously shift in the horizontal axis with a velocity of " $v / 2$ " in opposite directions, thus obtaining an equi-biaxial displacement (Fig. 2).

Specimens were mounted on the biaxial testing device inserting 12 hooks evenly distributed on the four sides of the specimen at a 5-mm distance from the edges. The spacing between hooks was $5 \mathrm{~mm}$. The specimen mounting procedure was aided by a custom made 
Table 1: Number of specimens tested for each donor. "Control" stands for the undecellularized specimens, while "HADM" indicates the Human Acellular Dermal Matrix specimens obtained after 5-week decellularization treatment.

\begin{tabular}{|c|c|c|}
\hline Donor & Control & HADM \\
\hline A & 4 & 4 \\
\hline B & 2 & 4 \\
\hline C & 4 & 4 \\
\hline
\end{tabular}

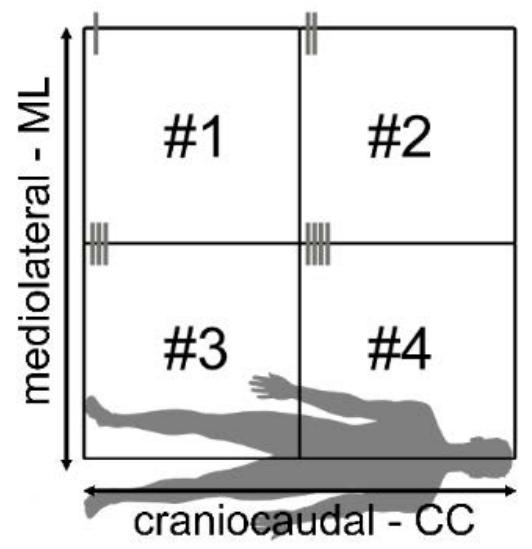

Figure 1: Specimens cutting and orientation with respect to the body. CC: craniocaudal direction; ML: medio-lateral direction.

a)

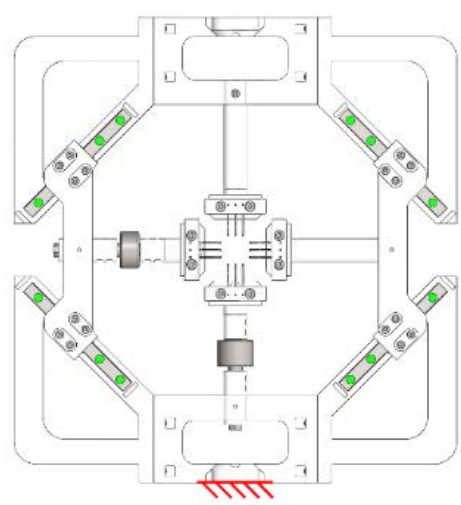

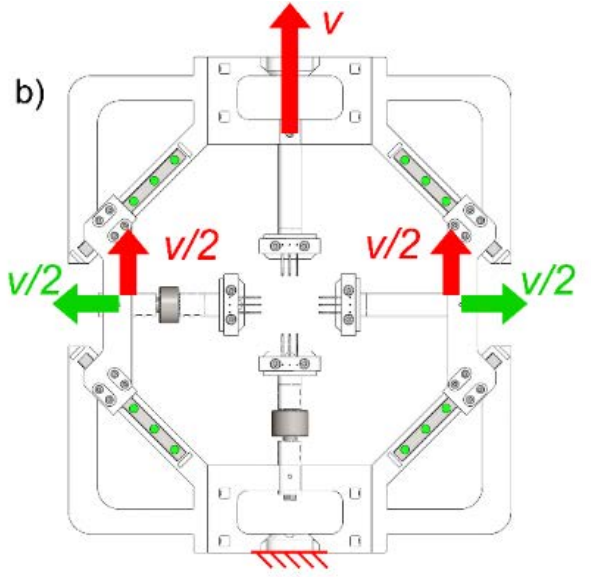

Figure 2: Biaxial device in a) close and b) open configuration. Red arrows represent vertical velocities whereas green arrows represent horizontal velocities. 

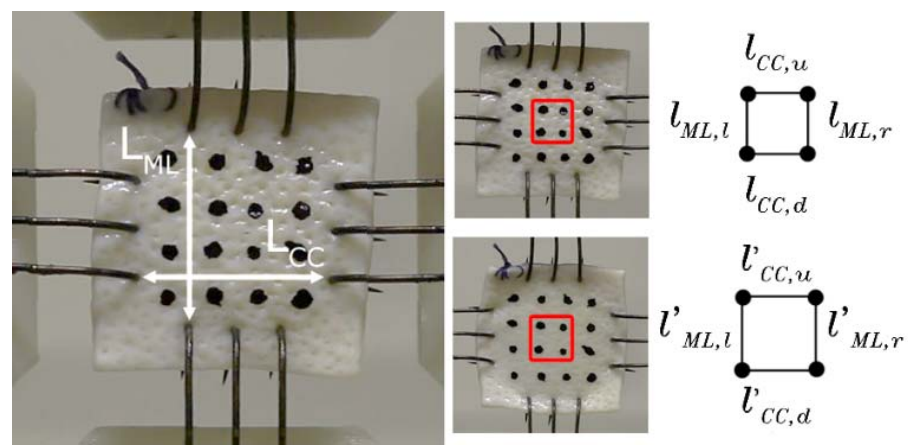

Figure 3: Specimen positioning into the biaxial device and parameters extraction for stress and strain evaluation. The red square encloses the four markers tracked for strain evaluation; $l_{i, j}$ stans for length along $i$ direction, on the $j$ specimen side ( $j=1$ for left; ' 'r' for right, ' $u$ ' for up; 'd' for down).

accessory kit for the simple and repeatable positioning of the biological specimen. A $4 \times 4$ matrix of markers was then drawn on the visible surface with an alcohol-based permanent black ink and afterwards the specimen was carefully positioned into the biaxial testing device (Fig. 3). The test speed was set to $0.16 \mathrm{~mm} / \mathrm{s}$ and the test was stopped at the achievement of a 10-mm displacement on each axis. The previously described digital camera was used to capture images of the specimen during the test with a $1 \mathrm{~Hz}$ frame rate.

\section{DATA ELABORATION}

Loads were acquired by two force transducers (Honeywell Sensotec Sensors Model 31 Mid) positioned along the two loading directions, while the marker deformations were measured optically using the photogrammetry set-up.

True stresses were then calculated dividing the load cell readings by the cross-sectional area of the specimen, which was taken as the product of the gauge length in the two orthogonal directions $\left(\mathrm{L}_{\mathrm{ML}}, \mathrm{L}_{\mathrm{CC}}\right)$ and the thickness of the specimen, performed in real-time. Gauge lengths were evaluated through ImageJ image analysis software as an average of three measures of the distance between two opposite hooks (Fig. 3), orthogonally to the loading direction (eqns (1)-(2)).

$$
\begin{aligned}
& \sigma_{M L}(t)=\frac{F_{M L}(t)}{L_{C C}(t) \cdot s(t)} \\
& \sigma_{C C}(t)=\frac{F_{C C}(t)}{L_{M L}(t) \cdot s(t)}
\end{aligned}
$$

The strain of the specimen along the two loading directions was then determined from the increment of the distance between two opposite central markers of the specimen, in the horizontal and vertical directions (eqns (3)-(4)). Markers centroids and trajectories were identified by a segmentation process performed in MATLAB environment.

$$
\begin{gathered}
\varepsilon_{M L}(t)=\operatorname{mean}\left(\frac{l_{M L, l}^{\prime}(t)-l_{M L, l}}{l_{M L, l}} ; \frac{l_{M L, r}^{\prime}(t)-l_{M L, r}}{l_{M L, r}}\right), \\
\varepsilon_{C C}(t)=\operatorname{mean}\left(\frac{\mathrm{l}_{\mathrm{CC}, \mathrm{u}}^{\prime}(\mathrm{t})-\mathrm{l}_{\mathrm{CC}, \mathrm{u}}}{\mathrm{l}_{\mathrm{CC}, \mathrm{u}}} ; \frac{\mathrm{l}_{\mathrm{CC}, \mathrm{d}}^{\prime}(\mathrm{t})-\mathrm{l}_{\mathrm{CC}, \mathrm{d}}}{\mathrm{l}_{\mathrm{CC}, \mathrm{d}}}\right),
\end{gathered}
$$


where:

- $\quad \mathrm{L}_{\mathrm{ML}}(\mathrm{t})$ and $\mathrm{L}_{\mathrm{CC}}(\mathrm{t})$ are the mean distances between two opposite hooks insertion points in the ML and in the CC direction respectively;

- $\quad \mathrm{F}_{\mathrm{ML}}(\mathrm{t})$ and $\mathrm{F}_{\mathrm{CC}}(\mathrm{t})$ are the ML and $\mathrm{CC}$ load cells outputs;

- $\mathrm{S}(\mathrm{t})$ is the specimen thickness over time, evaluated from $\mathrm{L}_{\mathrm{ML}}$ and $\mathrm{L}_{\mathrm{CC}}$ arrays and assuming an isochoric condition.

All parameters are depicted in Fig. 3. The slope of the toe region was evaluated considering 0 to $10 \%$ strain range on experimental curves so obtained.

\section{RESULTS AND DISCUSSION}

A representative set of results of biaxial tensile tests can be found in Fig. 4. It shows the stress-strain curves of four HADM specimens coming from the same donor (A). The graphs have been positioned according to specimen position in Fig. 1. In some cases, output data are not complete due to experimental errors: this is the case of untreated specimens coming from donor $\mathrm{B}$ where data from medio-lateral direction are missing.

Results underline the anisotropic behavior of dermis: in most cases, since the mechanical response changes significantly according to loading direction, stiffness along medio-lateral direction was higher than along cranio-caudal direction. This can be explained by the predominant orientation of collagen fibers that is aligned along the medio-lateral direction for dermis tissue taken from the lower back, in accordance with Langer findings and later works. Similar findings were described by Lanir and Fung [25] regarding the rabbit skin, and on other tissues. They found a stiffer behavior of the tissue along the body width (mediolateral direction) than along the body length, when biaxially stressed. The high material anisotropy causes a not homogenous strain distribution along the specimen, also affecting its central ROI (Region of Interest), in which the characteristic curve of the material is evaluated. Previous studies assessed the percentage of the area which can be considered to undergo a uniform strain distribution. Humphrey et al. [35] reported a uniform strain distribution within the central $4 \%$ of the specimen area, while Billiar and Sacks [27]found a uniformity of strains in the central $25 \%$ of the specimen area, having specified that they tested a homogeneous isotropic material with point loads at four discrete locations per side. It can be expected that the stress-strain distribution would be less uniform in an anisotropic tissue, even though direct experimental evidence would be needed. Nevertheless, Oomens et al. [36] claim that a homogeneous strain field cannot be obtained because of the inhomogeneous material properties and the challenging (sometimes impossible) manufacturing of sample. Anyhow, in this work about the 5\% of the gauge area (from hooks to hooks) was considered for the stress-strain curves extraction.

The slope of the toe region in a physiological strain range $(0-10 \%)$ was evaluated from each experimental curve. Results are shown in Fig. 5 and are reported in Table 2.

The higher stiffness of the tissue in the medio-lateral direction (along Langer lines) is here confirmed, with standard deviations relatively limited. The only exception is donor B tissue which shows generally much higher average values of elastic modulus, and high standard deviations along the medio-lateral direction in the decellularized tissues $(3.976 \pm 4.079 \mathrm{MPa})$. The mean elastic modulus for the medio-lateral direction is $43 \%$ to $74 \%$ higher than the cranio-caudal one, except for the decellularized tissue coming from donor $\mathrm{C}$ which shows a cranio-caudal elastic modulus $20 \%$ higher than the medio-lateral one. 

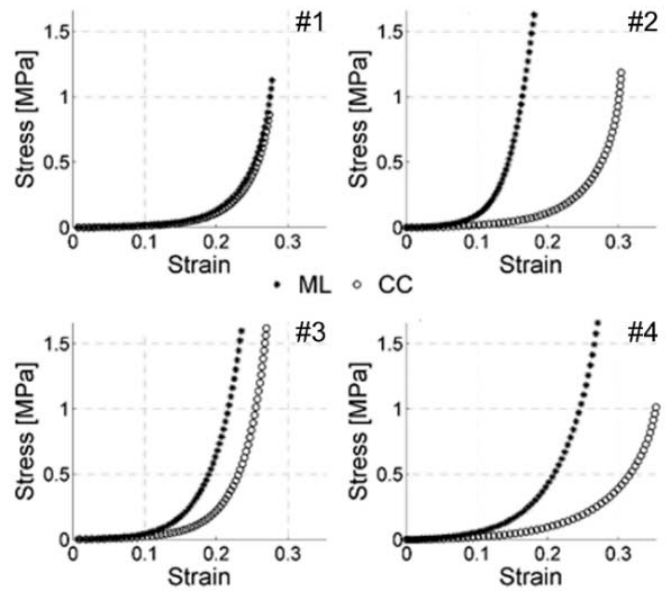

Figure 4: A representative set of stress-strain curves (HADM obtained from donor A). Plots have been positioned according to Fig. 1.

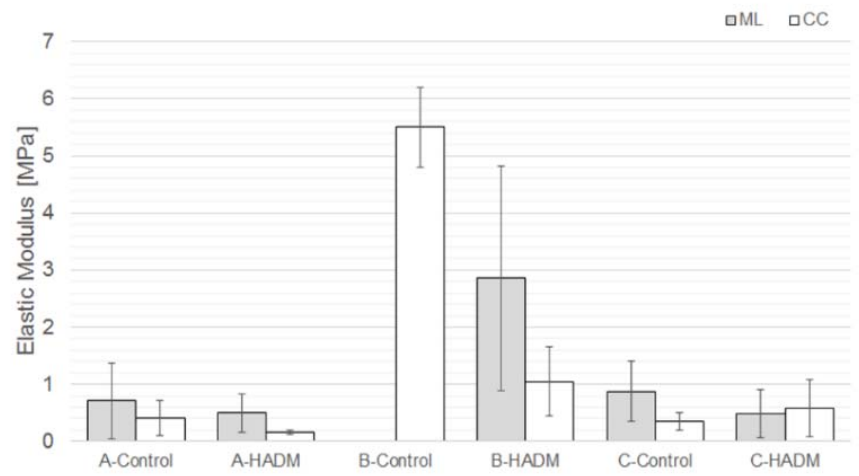

Figure 5: Biaxial elastic modulus for the medio-lateral and the cranio-caudal directions. Values are the average values \pm the standard deviation of the mean.

Table 2: Biaxial elastic modulus for the medio-lateral and the cranio-caudal directions (average $\pm \mathrm{SD}$ ).

\begin{tabular}{|c|c|c|c|}
\cline { 3 - 4 } \multicolumn{2}{c|}{} & \multicolumn{2}{c|}{ Elastic Modulus [MPa] (average \pm SD) } \\
\cline { 3 - 4 } \multicolumn{2}{c|}{} & Control & HADM \\
\hline \multirow{2}{*}{$\mathrm{A}$} & ML & $0.708 \pm 0.688$ & $0.496 \pm 0.335$ \\
\cline { 2 - 4 } & CC & $0.406 \pm 0.309$ & $0.163 \pm 0.038$ \\
\hline \multirow{3}{*}{$\mathrm{B}$} & ML & & $2.861 \pm 1.967$ \\
\cline { 2 - 4 } & CC & $5.506 \pm 0.699$ & $1.049 \pm 0.603$ \\
\hline \multirow{2}{*}{$\mathrm{C}$} & ML & $0.872 \pm 0.530$ & $0.481 \pm 0.424$ \\
\cline { 2 - 4 } & CC & $0.349 \pm 0.148$ & $0.582 \pm 0.507$ \\
\hline
\end{tabular}


Comparing the paired native and decellularized groups of specimens, the reduction of the mechanical properties of the treated dermis with respect to the native tissue is here again confirmed for all donors, for both directions. Therefore, the missing medio-lateral results for donor B let envisage that a mean elastic modulus higher than 5.5 MPa would be obtained.

Surgical procedures begin and end with incisions, and the incisions planning is a mandatory step when a good outcome is desired in terms of scars and tension distribution in the scar proximity. The incision planning is intimately related to the Langer's lines orientation, because incisions made parallel to Langer's lines may heal better and produce less scarring than incisions made across Langer's lines. This consideration can also be transferred to cases which do not directly involve aesthetics, such as soft tissues reconstructive surgeries, were a biological graft is needed. When placed in a body part where the substitute will bear a physiological and anisotropic distribution of stress, the orientation of the graft should be consistent with the surrounding tissue. Therefore, not only the stiffer direction of the host tissue should be known, but also the substitute one and, ideally, an equi-biaxial test performed on a small square specimen excerpts from the graft could provide this indication.

\subsection{Conclusions}

The biaxial mechanical behavior of native dermis and HADM was measured exploiting a purpose-made biaxial conversion device. Stress-strain curves were evaluated from loads acquired by two load cells positioned along two orthogonal axes and the optical measure of the displacements of four markers located in the central area of the specimen. Comparisons among the native and decellularized tissues demonstrated the loss of mechanical strength caused by the decellularization treatment. Moreover, the specimens resulted, on average, less extensible in the medio-lateral direction (namely, along the Langer lines) than in the craniocaudal direction, confirming the correlation of dermis mechanical response with collagen fibers orientation with respect to the loading direction.

\section{REFERENCES}

[1] Shi, L.J., Wang, J., Yang, C. \& Jiang, W.W., Application of Acellular Dermal Matrix in Reconstruction of Oral Mucosal Defects in 36 Cases. Journal of Oral and Maxillofacial Surgery, 70(11), pp. 586-591, 2012.

[2] Duncan, D.I., Correction of implant rippling using allograft dermis. Aesthetic Surgery Journal, 21(1), pp. 81-84, 2001.

[3] Breuing, K.H. \& Warren, S.M., Immediate bilateral breast reconstruction with implants and inferolateral AlloDerm slings. Annals of Plastic Surgery, 55(3), pp. 232239, 2005.

[4] Garvey, P.B., Martinez, R.A., Baumann, D.P., Liu, J. \& Butler, C.E., Outcomes of Abdominal Wall Reconstruction with Acellular Dermal Matrix Are Not Affected by Wound Contamination. Journal of the American College of Surgeons, 219(5), pp. 853864, 2014.

[5] Barber, F.A., Herbert, M.A. \& Boothby, M.H., Ultimate tensile failure loads of a human dermal allograft rotator cuff augmentation. Arthroscopy: The Journal of Arthroscopic and Related Surgery, 24(1), pp. 20-24, 2008.

[6] Burkhead, W.Z., Schiffern, S.C. \& Krishnan, S.G., Use of Graft Jacket as an Augmentation for Massive Rotator Cuff Tears. Seminars in Arthroplasty, 18(1), pp. 11-18, 2007. 
[7] Snyder, S.J. \& Bond, J.L., Technique for Arthroscopic Replacement of Severely Damaged Rotator Cuff Using "GraftJacket” Allograft. Operative Techniques in Sports Medicine, 15(2), pp. 86-94, 2007.

[8] Wong, I., Burns, J. \& Snyder, S., Arthroscopic GraftJacket repair of rotator cuff tears. Journal of Shoulder and Elbow Surgery. 19(2S), pp. 104-109, 2010.

[9] Lee, D.K., Achilles Tendon Repair with Acellular Tissue Graft Augmentation in Neglected Ruptures. The Journal of Foot and Ankle Surgery, 46(6), pp. 451-455, 2007.

[10] Barber, F.A., Herbert, M.A. \& Coons, D.A., Tendon Augmentation Grafts: Biomechanical Failure Loads and Failure Patterns. Arthroscopy: The Journal of Arthroscopic and Related Surgery, 22(5), pp. 534-538, 2006.

[11] Moore, M.A., Samsell, B., Wallis, G., Triplett, S., Chen, S., Jones, A.L. et al., Decellularization of human dermis using non-denaturing anionic detergent and endonuclease: a review. Cell and Tissue Banking, 16(2), pp. 249-259, 2015.

[12] Nì Annaidh, A., Bruyère, K., Destrade, M., Glichrist, M.D. \& Otténio, M., Characterization of the anisotropic mechanical properties of excised human skin. Journal of the Mechanical Behavior of Biomedical Materials, 5, pp. 138-148, 2012a.

[13] Waldman, S.D. \& Lee, J.M., Boundary conditions during biaxial testing of planar connective tissues. Part 1: Dynamic Behavior. Journal of Materials Science: Materials in Medicine, 13(10), pp. 933-938, 2002.

[14] Skulborstad, A.J., Swartz, S.M. \& Goulbourne, N.C., Biaxial mechanical characterization of bat wing skin. Bioinspiration \& Biomimetics, 10(3), 2015.

[15] Grashow, J.S., Yoganathan, A.P. \& Sacks, M.S., Biaixal stress-stretch behavior of the mitral valve anterior leaflet at physiologic strain rates. Annals of Biomedical Engineering, 34(2), pp. 315-325, 2006.

[16] Zemánek, M., Burša, J. \& Děták, M., Biaxial Tension Tests with Soft Tissues of Arterial Wall. Engineering Mechanics, 16(1), pp. 3-11, 2009.

[17] Prendergast, P.J., Lally, C., Daly, S. \& Reid, A.J., Analysis of Prolapse in Cardiovascular Stents: A Constitutive Equation for Vascular Tissue and FiniteElement Modelling. Journal of Biomechanical Engineering, 125(5), pp. 692-699, 2003.

[18] Lally, C., Reid, A.J. \& Prendergast, P.J., Elastic behavior of porcine coronary artery tissue under uniaxial and equibiaxial tension. Annals of Biomedical Engineering, 32(10), pp. 1355-1364, 2004.

[19] Kahlon, A., Hurtig, M.B. \& Gordon, K.D., Regional and depth variability of porcine meniscal mechanical properties through biaxial testing. Journal of Mechanical Behaviour of Biomedical Materials, 41, pp. 108-114, 2015.

[20] Sacks, M.S., Biaxial Mechanical Evaluation of Planar Biological Materials. Journal of Elasticity, 61, pp. 199-246, 2001.

[21] Brieu, M., Diani, J. \& Bhatnagar, N., A New Biaxial Tension Test Fixture for Uniaxial Testing Machine - A Validation for Hyperelastic Behavior of Rubber-like Materials. Journal of Testing and Evaluation, 35(4), 2007.

[22] Cavallaro, P., Sadegh, A., Quigley, C. \& Johnson, A., Effects of Coupled Biaxial Tension and Shear Stresses on Decrimping in Pressurized Woven Fabrics. NUWCNPT Technical Report, 11(571), 2004.

[23] Duncan, B., Test methods for determining hyperelastic properties of flexible adhesives. NPL Report CMMT(MN)054, 1999.

[24] Lanir, Y. \& Fung, Y.C., Two-dimensional mechanical properties of rabbit skin - I. Experimental system. Journal of Biomechanics, 7(1), pp. 29-34, 1974a.

[25] Lanir, Y. \& Fung, Y.C., Two-dimensional mechanical properties of rabbit skin - II. Experimental results. Journal of Biomechanics, 7(2), pp. 171-182, 1974b. 
[26] Tong, P. \& Fung, Y.C., The stress strain relationship for the skin. Journal of Biomechanics, 9(10), pp. 649-657, 1976.

[27] Billiar, K.L. \& Sacks, M.S., Biaxial Mechanical Properties of the Natural and Glutaraldehyde Treated Aortic Valve Cusp - Part I: Experimental Results. Journal of Biomechanical Engineering, 122, pp. 23-30, 2000.

[28] Maiti, R. et al., In vivo measurement of skin surface strain and sub-surface layer deformation induced by natural tissue stretching. Journal of the Mechanical Behavior of Biomedical Materials, 42, pp. 556-569, 2016.

[29] Terzini, M., Bignardi, C., Castagnoli, C., Cambieri, I., Zanetti, E.M. \& Audenino, A.L., Dermis mechanical behaviour after different cell removal treatments. Medical Engineering \& Physics, 38(9), pp. 862-869, 2016.

[30] Terzini, M., Bignardi, C., Castagnoli, C., Cambieri, I., Zanetti, E.M. \& Audenino, A.L., Ex vivo dermis mechanical behavior in relation to decellularization treatment length. Open Biomedical Engineering Journal. 10, pp. 34-42, 2016.

[31] Langer, K., On the anatomy and physiology of the skin. I. The cleavability of the cutis. British Journal of Plastic Surgery, 31(1), pp. 3-8, 1978.

[32] Yoder, J.H. \& Elliott, D.M., Nonlinear and anisotropic tensile properties of graft materials used in soft tissue applications. Clinical Biomechanics ,25(4), pp. 378-382, 2010.

[33] Zanetti, E.M., Perrini, M., Bignardi, C. \& Audenino, A.L., Bladder tissue passive response to monotonic and cyclic loading. Journal of Biorheology, 49(1), pp. 49-63, 2012.

[34] Natali, A.N., Audenino, A.L., Artibani, W., Fontanella, C.G., Carniel, E.L. \& Zanetti, E.M., Bladder tissue biomechanical behavior: experimental tests and constitutive formulation. Journal of Biomechanics, 48, p. 3088-3096, 2015.

[35] Humphrey, J.D., Vawter, D.L. \& Vito, R.P., Quantification of strains in biaxially tested soft tissues. Journal of Biomechanics, 20(1), pp. 59-65, 1987.

[36] Oomens, C.W., van Ratingen, M.R., Janssen, J.D., Kok, J.J. \& Hendriks, M.A., A Numerical-Experimental Method for a Mechanical Characterization of Biological Materials. Journal of Biomechanics, 26(4/5), pp. 617-621, 1993.

[37] Manschot, J.F.M. \& Brakkee, A.J.M., The Measurement and Modelling of the Mechanical Properties of Human Skin in vivo - I. The Measurement. Journal of Biomechanics, 19(7), pp. 511-515, 1986.

[38] Waldorf, J.C., Perdikis, G. \& Terkonda, S.P. Planning Incisions. Operative Techniques in General Surgery, 4(5), pp. 199-206, 2002. 